HIE. Our results confirm the potential utility of miRNA biomarkers in the early diagnosis of HIE.

\section{PS-152 ACTIVIN-A: A BIOMARKER OF SEVERE ENCEPHALOPATHY}

AM Looney, BH Walsh, NL Denihan, GB Boylan, DM Murray. The Neonatal Brain Research Group Irish Centre for Fetal and Neonatal Translational Research (INFANT) Department Paediatrics and Child Health, University College Cork, Cork, Ireland

\subsection{6/archdischild-2014-307384.448}

Background Hypoxicischaemic encephalopathy (HIE) remains one of the leading causes of neonatal morbidity and mortality. Therapeutic hypothermia may improve the outcome of infants with moderate/severe encephalopathy but only if initiated within six hours of the initial insult. The aim of our study was to determine if umbilical cord blood (UCB) levels of Activin-A, a glycoprotein previously implicated in neuronal brain injury, could identify infants with moderate/severe encephalopathy at birth.

Methods Full term infants with perinatal asphyxia (PA) were identified by a cord $\mathrm{pH}<7.1$ and/or five minute Apgar score $\leq$ 6 and/or requirement for intubation/CPR at birth. Following diagnosis at delivery, UCB was drawn, processed and bio-banked. HIE grade was confirmed with early continuous EEG monitoring and modified Sarnat score. Activin-A analysis was carried out using ELISA (R\&D Systems).

Results In total 156 infants (controls $=78$, cases $=78$ ) were included in the study. Cases included 56 infants with PA (nonHIE) and 24 infants with HIE (mild $=14$, moderate $=6$, severe $=4$ ). Following analysis, a significant increase in Activin-A expression was observed between the control and severe HIE groups, and between the perinatal asphyxia and severe HIE groups (median $(\mathrm{SD})=487.48(470.21)$ vs $911.54(594.1) \mathrm{p}=$ 0.032 and 487.95 (384.1) vs 911.54 (594.1), $\mathrm{p}=0.035$, respectively). No significant difference was seen between PA and mild or moderate HIE. Using a cut-off value of $724.5 \mathrm{pg} / \mathrm{ml}$ we report Activin-A has a $100 \%$ negative predicitive value, with a sensitivity and specificity of $100 \%$ and $70 \%$ respectively.

Conclusion Our study supports the use of Activin-A as a biomarker of severe encephalopathy.

\section{PS-153 REFERENCE VALUES OF MALONDIALDEHYDE IN BLOOD AND URINE OF THE HEALTHY TERM NEWBORN IN THE PERINATAL PERIOD}

${ }^{1}$ A Cilla, ${ }^{2} \mathrm{~J}$ Arnaez, ${ }^{3} \mathrm{P}$ Muñiz, ${ }^{4} \mathrm{M}$ Cavia, ${ }^{4} \mathrm{R}$ Alcaraz, ${ }^{1} \mathrm{~L}$ Puente, ${ }^{1} \mathrm{G}$ Aja, ${ }^{1} \mathrm{~N}$ Gorria. ${ }^{1}$ Pediatrics, Hospital Universitario de Burgos, Burgos, Spain; ${ }^{2}$ Neonatology Pediatrics, Hospital Universitario de Burgos, Burgos, Spain; ${ }^{3}$ Faculty of Sciences, Universidad de Burgos, Burgos, Spain; ${ }^{4}$ Research Unit, Hospital Universitario de Burgos, Burgos, Spain

\subsection{6/archdischild-2014-307384.449}

Introduction A certain amount of oxidative stress has a role in the normal progression of embryonic and fetal growth, as well as during labour. In contrast, increased OS has been involved in the causation or worsening of several gestational, fetal and neonatal diseases. Cell lipid peroxidation by free radicals causes membrane lipid disruption and is potentially harmful. Malondialdehyde (MDA) is one of the end products of lipid peroxidation, which can be interpreted as a marker of the extent of damage to cells and the anti-oxidative system capacity.

Objective As part of a study on oxidative stress on the term newborn, we aimed to determine the baseline levels of MDA in blood and urine of healthy term newborns.
Patients and methods All newborns above 35 gestational weeks born in our institution from October 2012 - March 2013 were eligible for study. Newborns with potential risk factors for increased oxidative stress were excluded for this analysis. Blood samples at birth (cord arterial and venous blood) and at $48 \mathrm{~h}$ postnatal life (heel puncture) were collected. Urine from the first and second day was collected.

Results 204 newborns (90 females and 114 males) met the inclusion criteria. Reference MDA levels were as follows $(\mu \mathrm{M}$, mean \pm SD): cord vein $3.37 \pm 1.16$; cord artery $3.33 \pm 0.94$. At $48 \mathrm{~h}$ postnatal life, $3.29 \pm 0.91$. Urinary levels were as follows: first day urine $1.24 \pm 0.87$; second day urine $1.48 \pm 0.99$. There were no statistical differences between males and females.

Conclusions These are data from a large group of newborns, aiming to give an accurate description of the baseline levels of malondialdehyde in our population, which could be useful when it comes to making therapeutic decisions in the future.

\section{PS-154 HAS THERAPEUTIC HYPOTHERMIA (TH) CHANGED THE PROGNOSTIC VALUE OF CLINICAL EVALUATION OF NEONATAL HYPOXIC-ISCHAEMIC ENCEPHALOPATHY (HIE)? A SYSTEMATIC REVIEW AND META-ANALYSIS}

${ }^{1}$ A Alarcon Allen, ${ }^{2} \mathrm{C}$ Ochoa, ${ }^{3} \mathrm{R}$ del Rio, ${ }^{4} \mathrm{~J}$ Gonzalez de Dios, ${ }^{5} \mathrm{~J}$ Arnaez, ${ }^{6} \mathrm{G}$ Arca, ${ }^{7} \mathrm{~A}$ Balaguer, ${ }^{3} \mathrm{~A}$ Garcia-Alix, ${ }^{8}$ Working Group for the National Health System Clinical Practice Guideline on Neonatal Hypoxic-Ischaemic Encephalopathy. ${ }^{1}$ Neonatal Unit, Oxford University Hospitals NHS Trust, Oxford, UK; ${ }^{2}$ Service of Paediatrics, Complejo Asistencial de Zamora, Zamora, Spain; ${ }^{3}$ Neonatal Unit Hospital Sant Joan de Deu, Agrupacio Sanitaria Sant Joan de Deu-Hospital Clinic Universitat de Barcelona, Barcelona, Spain; ${ }^{4}$ Department of Paediatrics, Hospital General Universitario de Alicante Universidad Miguel Hernandez, Alicante, Spain; ${ }^{5}$ Neonatal Unit, Hospital Universitario de Burgos, Burgos, Spain; ${ }^{6}$ Neonatal Unit Hospital Clinic, Agrupacio Sanitaria Sant Joan de DeuHospital Clinic Universitat de Barcelona, Barcelona, Spain; 7 Department of Paediatrics, Hospital General de Catalunya Universitat Internacional de Catalunya, Barcelona, Spain; ${ }^{8}$ Spain

\subsection{6/archdischild-2014-307384.450}

Background Clinical grading of HIE correlates with outcome. TH improves survival and neurodevelopment in HIE. Aim: To review the effect of $\mathrm{TH}$ on the prognostic value of clinical grading of HIE and its course.

Methods Systematic review and meta-analysis of studies on the ability of Sarnat stage at defined times to predict death/disability at $\geq 18 \mathrm{~m}$ in normothermia and TH-treated term neonates with HIE. Pooled risks were estimated, with random effect models, according to HIE stage and treatment.

Results Data on encephalopathy stage at $<6 \mathrm{~h}$ were available from seven TH trials including 1214 newborns with moderate/severe HIE. Post-hoc studies of two trials (381 infants) provided $72 \mathrm{~h}$ data.

The proportion of infants with moderate encephalopathy at $<6 \mathrm{~h}$ who had poor outcome was 52\% (95\% CI:44-60; $\mathrm{I}^{2}=$ $48 \%)$ in normothermia-treated and 35\% (95\% CI:28-41; $\mathrm{I}^{2}=$ $51 \%)$ in TH-treated neonates. The proportion for severe encephalopathy was 83\% (95\% CI:72-93; $\left.\mathrm{I}^{2}=81 \%\right)$ in normothermia and 67\% (95\% CI:58-76; $\left.\mathrm{I}^{2}=74 \%\right)$ in TH. At $<6 \mathrm{~h}$, the OR for severe vs moderate HIE to predict unfavourable outcome was $4.14\left(95 \% \mathrm{CI}: 2.40-7.13 ; \mathrm{I}^{2}=35 \%\right)$ in normothermia and 3.77 (95\% CI:2.62-5.41; $\mathrm{I}^{2}=0 \%$ ) in $\mathrm{TH}$.

$\mathrm{TH}$ did not affect HIE grade at $72 \mathrm{~h}$. No improvement of encephalopathy at $72 \mathrm{~h}$ increased the risk of poor outcome (OR 8.21, 95\% CI:2.01-33.6; $\mathrm{I}^{2}=74 \%$ ). The ORs for persistent moderate and severe encephalopathy at $72 \mathrm{~h}$ to predict unfavourable outcome were 5.09 (95\% CI:1.53-16.92; $\mathrm{I}^{2}=66 \%$ ) and 42.83 (95\% CI:13.55-135.37; $\left.\mathrm{I}^{2}=44 \%\right)$. 